\title{
Psychopathy-Related Differences in Selective Attention Are Captured by an Early Event-Related Potential
}

\author{
Arielle Baskin-Sommers, John J. Curtin, Wen Li, and Joseph P. Newman \\ Department of Psychology, University of Wisconsin.
}

\begin{abstract}
According to the response modulation model, the poorly regulated behavior of psychopathic individuals reflects a problem reallocating attention to process peripheral information while engaged in goal-directed behavior (Patterson \& Newman, 1993). We evaluated this tenet using male prisoners and an early event-related potential component (P140) to index attentional processing. In all task conditions, participants viewed and categorized letter stimuli that could also be used to predict electric shocks. Instructions focused attention either on the threat-relevant dimension of the letters or an alternative, threat-irrelevant dimension. Offenders with high scores on Hare's (2003) Psychopathy Checklist-Revised displayed a larger P140 under alternative versus threat conditions. Beyond demonstrating psychopathy-related differences in early attention, these findings suggest that psychopathic individuals find it easier to ignore threat-related distractors when they are peripheral versus central to their goal-directed behavior.
\end{abstract}

\section{Keywords}

psychopathy; ERP; attention

\begin{abstract}
Psychopathic individuals are characterized by difficulty establishing genuine relationships, superficial affective experiences, an impulsive behavioral style, and a chronic antisocial lifestyle that entails great costs to society as well as for the affected individual (e.g., incarceration). Although psychopathy is associated with an array of affective, inhibitory, and language deficits (e.g., Kiehl, 2006), etiological explanations for the disorder typically emphasize their affective deficits (Blair \& Mitchell, 2009; Blair et al., 1995; Lykken, 1957; Patrick, 1994; Patrick, Bradley \& Lang, 1993). However, such accounts commonly undervalue the role that cognitive-affective interactions have in modulating the etiological and phenotypic manifestations of psychopathy.
\end{abstract}

According to Newman and colleagues' response-modulation model, an early attention bottleneck plays a crucial role in moderating the affective, behavioral, and decision-making deficits associated with psychopathy. This attention bottleneck stems from models of selective attention in which there is a continuum of early and late influences. Early selective attention is described as a "fixed bottleneck" that, once established, blocks the processing of peripheral information that is not goal relevant (Driver, 2001). Such selection can affect neural activity in the visual cortex (Kastner \& Ungerleider, 2000), filter the processing of sensory information (Hillyard, Vogel, \& Luck, 1998), and prevent the perception of secondary information (Lavie et al., 2004). Alternatively, selective attention may operate at 
a later stage, as a function of memory and response selection based on top-down goals (Deutsch \& Deutsch, 1963; Duncan, 1980). With regard to psychopathic individuals, evidence suggests that they are associated with attentional dysfunction at the early, bottleneck stage of selection (Baskin-Sommers, Curtin \& Newman, 2011; Newman \& Baskin-Sommers, in press).

Specifically, psychopaths are oblivious to potentially meaningful peripheral information because once engaged in goal-directed behavior they fail to reallocate attention to other potentially meaningful information (MacCoon et al., 2004; Newman, 1998; Patterson \& Newman, 1993). This difficulty balancing demands to process goal-directed and peripheral information is believed to create a bias whereby psychopaths are unresponsive to information unless it is a central aspect of their goal-directed focus of attention (Hiatt \& Newman, 2004; Jutai \& Hare, 1983; Kiehl, Hare, McDonald, \& Brink, 1999).

To date, research on this proposal has manipulated task focus, while measuring behavior, skin conductance, and/or fear-potentiated startle (FPS) to evaluate this supposition. However, there has yet to be a direct substantiation of the posited early selective attention mechanism. Event-related potentials (ERPs) are especially well suited to quantifying and exploring such attentional processing (Luck \& Hillyard, 1999).

In the field of psychopathy there has been a limited number of ERP studies (Jutai \& Hare, 1983; Jutai, Hare, \& Connolly, 1987; Forth \& Hare, 1989; Raine \& Venable, 1989; Williamson, Harpur, \& Hare, 1991; Kiehl, Hare, Mc-Donald, \& Liddle, 1999; Kiehl, Smith, Hare, \& Liddle, 2000; Flor et al., 2002; Kiehl, Bates, Laurens, Hare, \& Liddle, 2006). The preponderance of these studies has focused on N100 (Jutai \& Hare, 1983), P300 (Flor et al., 2002; Kiehl et al., 2006), and late slow wave components (e.g., contingent negative variation; Raine \& Venable, 1989). Unfortunately, the results of these studies allow few firm conclusions owing, in large part, to the heterogeneity of the participants and the variety of tasks employed (e.g., oddball, S1-S2 motor response, and aversive differential conditioning tasks). Despite these inconsistencies in the ERP-psychopathy literature, a common interpretation of the significant findings is that they indicate a potential psychopathy-related difficulty with attention, orienting, processing efficiency, and monitoring of sequential information (Kiehl et al., 2006). Of particular relevance to the response modulation model, Jutai and Hare (1983) reported preliminary evidence that psychopathic individuals "overfocus" attention on goal-relevant activities, resulting in smaller N100 responses to irrelevant tone pips. Such findings suggest that the affective deficits of psychopathic individuals may also vary as a function of attentional focus. However, no study has directly examined psychopathy-related differences in early attentional ERPs using a paradigm that involves significant emotion cues and an attentional manipulation of the type specified by the response modulation model.

In this study, we begin to characterize the selective attention abnormalities associated with psychopathic individuals' diminished response to peripheral stimuli using electrophysiological data (i.e., ERP) collected by Newman, Curtin, Bertsch, and BaskinSommers (2010). Newman and colleagues used a task that required participants to view and categorize letter stimuli, which also could be used to predict electric shocks. Instructions were used to activate goal-directed behavior that involved either a focus on threat-relevant information (i.e., the color which predicted electric shocks) or focused attention on an alternative, threat-irrelevant dimension of the letter stimuli (i.e., upper/lower case of letter, match/mismatch in a two-back task). Psychopathy scores were significantly and inversely related to FPS under conditions that required participants to focus on a threat-irrelevant dimension of stimuli. In contrast, psychopathy scores were unrelated to FPS under conditions that focused attention on the threat-relevant dimension. 
Paralleling the results for FPS reported by Newman et al. (2010), we predicted that the psychopathy-early ERP association postulated by the response modulation model would differ depending upon whether instructions directed attention to a threat-relevant dimension of the stimuli or an alternative focus on threat-irrelevant information. More specifically, we were interested in locating an ERP window that might be interpreted as a feed-forward processes related to early selective attention (i.e., within $200 \mathrm{~ms}$ of stimulus onset; Lamme $\&$ Roelfsema, 2000). We predicted that psychopathy scores would be associated with larger ERP (i.e., greater early selection) in the alternative-focus versus threat-focus conditions. This prediction is predicated on the assumption that the response modulation deficit of psychopathic individuals results in a rigid prepotent focus on primary task features that facilitates ignoring peripheral threat information under alternative-focus conditions. Conversely, for individuals low on psychopathy, we assumed that a bias to "answer the call for processing signaled by unexpected or threatening peripheral information" (Patterson \& Newman, 1993) would influence attention regardless of whether the threat cues were central or peripheral to the goal-relevant focus of attention.

\section{Methods}

Participants

Participants were 101 European American male inmates from a maximum security prison in Southern Wisconsin. Participants in the present sample were a subset of the sample of 125 inmates in the Newman et al. FPS study (2010). From the 125 participants in Newman et al., additional participants were excluded due to data loss on critical ERP channels $(N=21)$ and exceeding the threshold on artifact rejection $(N=3)$. Sample characteristics and descriptive statistics are presented in Table 1.

\section{Psychopathy Checklist-Revised (PCL-R; Hare, 2003)}

All participants were assessed using file information and a semistructured interview that lasted approximately $60 \mathrm{~min}$. The PCL-R contains 20 items that are rated according to the degree to which a characteristic is present: significantly (2), moderately (1), or not at all (0). In the present study, interrater reliability for the 12 participants with dual PCL-R ratings was .89 .

\section{Experimental Task (Newman et al., 2010)}

During the task, participants viewed a series of letter cues. The letter cues were either upperor lowercase and were colored either red or green. A participant's focus of attention varied depending upon which of the three conditions they were performing. There were 100 trials per condition and the condition order was fully counterbalanced across participants. In the threat-focus condition (TF), participants were instructed to attend to the color of the letter cue and press one of two buttons according to whether the letters indicated threat (red) or safety (green). This condition was designed to focus participants on the feature of the letter cue (i.e., color) that indicated threat of shock. In the alternative-focus/low-load condition (AF/LL) participants were asked to determine if the letter cue was upper- or lowercase. In the alternative-focus/ high-load condition (AF/HL), participants performed a two-back task, where they had to attend to each letter in a series in order to indicate if the current letter matched the letter presented two trials back in the series. The purpose of the alternativefocus conditions was to make the threat information secondary to the primary task (i.e., attend to letter case or identity). Evaluation of attentional focus is achieved by contrasting the condition that required participants to focus on the threat information (TF) with the other two threat-irrelevant focus conditions (AF/LL and $\mathrm{AF} / \mathrm{HL}$ ). Conversely, contrasting the two threat-irrelevant conditions serves to clarify the effects of working memory load. 
Participants were told that in all conditions, electric shocks might be administered on some trials following red letters (threat), but that no shocks would follow green letters (safety). Shocks occurred 1,400 ms poststimulus onset and were administered on $20 \%$ of threat trials in each condition, for a total of 30 shocks (see Newman et al. for shock procedure). Additionally, 48 startle-eliciting noise probes (50-ms, 102-dB white noise bursts) were presented 1,400 ms post-letter onset. 1

\section{EEG Procedures and Analysis}

Stimulus presentation and data collection were run on PC-based DMDX (Forster \& Forster, 2003) and Neuroscan Synamps 2 amplifiers and acquisition software (Compumedics, Charlotte, NC). EEG was recorded from $\mathrm{Ag}-\mathrm{AgCl}$ electrodes mounted in an elastic cap (Electro Cap International, Eaton, $\mathrm{OH}$ ) and located at standard midline positions (Fz, FCz, $\mathrm{Cz}$, and $\mathrm{Pz}$ ), plus two vertical eye-movement electrodes (VEOG; placed above and below the eye in line with the pupil). Electrode impedance for all channels was kept below $10 \mathrm{~K} \Omega$ by lightly abrading the scalp and applying gel in the sensor cups. All electrodes were referenced to the left mastoid electrode. Offline, signals were digitally filtered with a $15-\mathrm{Hz}$ low-pass filter and resampled at $500 \mathrm{~Hz}$. EEG signals were averaged for 300-ms periods starting $100 \mathrm{~ms}$ prior to stimulus onset. To exclude trials contaminated by artifacts, trials with a voltage exceeding $\pm 100 \mathrm{mV}$ at VEOG and $\pm 75 \mathrm{mV}$ at any of the midline sites, relative to the 100-ms baseline, were discarded before further analysis.

ERPs were averaged separately for all correct trials within each stimulus type (threat vs. safety) and task condition (TF, AF/LL, and AF/HL). A minimum of at least 30 trials in each condition was established, and an average of 36 (out of 50) trials remained in each category after artifact rejection. Again, our goal was to find the earliest possible component that tracked the condition manipulation in order to determine whether the psychopathy-related differences would manifest in an early ERP component. Inspection of the grand average ERP waveforms for all participants across all conditions (see Figure 1) revealed a positive potential that peaked at $140 \mathrm{~ms}$ (P140) post-stimulus onset. The magnitude of P140 was measured as the mean amplitude in the window of 115-165 ms poststimulus.2

\section{Data Analysis}

In order to examine the relationship between early ERP (P140) and psychopathy, we analyzed P140 in a general linear model (GLM), with task condition, Threat [present (red) or absent (green)] and electrode site as within-subject categorical factors and psychopathy total score (mean-centered and standardized) as a between-subject quantitative variable. Following Newman et al. (2010), we also ran tests of orthogonal interaction contrasts as a part of the GLM design. The first contrast examined the attention manipulation (e.g., TF vs. two AF conditions). The second interaction contrast compared the two alternative focus conditions to examine the effects of low versus high cognitive load on psychopathy (AF/LL vs. AF/HL).

\footnotetext{
${ }^{1}$ This task was initially analyzed with regard to startle responses, and thus the noise bursts that elicit startle responses were presented. Noise burst trials were included in the ERP analysis, but ERP responses to the noise bursts were not analyzed since the onset of the stimuli occurred well before the probes.

${ }^{2}$ In the absence of prior publications characterizing the ERPs associated with this new research paradigm, it was not possible to specify an a priori ERP component associated with early attentional processing in the context of this task. Rather, after examining the ERP characteristics for the general sample, we focused on an ERP window that was consistent with indexing early attentional processes (Lamme \& Roelfsema, 2000).
} 


\section{Results3}

Prior to our hypothesis testing, we examined the potential effect of scalp distribution (i.e., difference based on electrode site). The effect of electrode site on P140 magnitude was significant, such that P140 increased from the parietal to frontal sites, $F(3,297)=12.00, p$ $<.01$. Additionally, site significantly interacted with threat and condition, respectively (Site $\times$ Threat: $F(3,297)=9.42, p=.001$; Site $\times$ Condition: $F(6,594)=6.28, p<.01)$. However, in the absence of a three-way interaction among site, threat, and condition $(p=.747)$, as well as an interaction between site and psychopathy $(p=.689)$, we averaged across electrode site when exploring the relationships between threat, focus of attention, and psychopathy on P140 magnitude.

The main effect of psychopathy was not significant $(p=.909)$, indicating that psychopathy total scores were not consistently related to P140 when collapsing across other factors. Consistent with the response modulation model, the relationship between psychopathy and P140 was moderated by threat and focus of attention (i.e., Psychopathy $\times$ Threat $\times$ Attentional focus interaction contrast), $F(1,99)=5.024, p=.027$. To unpack the significant three-way interaction, we examined the effect of psychopathy on the attention manipulation (TF vs. AF) in the green and red trials (i.e., simple interaction effects at each level of threat). As displayed in Figure 2, individuals high on psychopathy, as compared with those low on psychopathy, had greater P140 in the AF conditions than the TF condition, particularly during presentation of red stimuli. The beta, reflecting the difference between $\mathrm{TF}$ and $\mathrm{AF}$ conditions, was significant for the red stimuli $(\mathrm{B}=-.46, p=.009)$, but did not approach significance for the green stimuli $(\mathrm{B}=.003, p=.98)$. A second interaction contrast examining the effects of high versus low cognitive load on psychopathy was not significant $(p=.950)$.

\section{Supplementary Analyses: Two-Factor Model of Psychopathy}

Some researchers advocate parsing psychopathy into two components (i.e., Factor 1 and Factor 2). Factor 1 reflects the interpersonal (charm, grandiosity, and deceitfulness/conning) and affective (lack of remorse, empathy, and emotional depth) features of psychopathy. Alternatively, Factor 2 describes the impulsive and chronic antisocial tendencies associated with psychopathy.

Analyzing the data within this framework reveals a replication of the psychopathy total score effect in individuals high on Factor 1 [omnibus: $F(2,196)=3.53, p=.032, \eta_{\mathrm{p}}^{2}=.035$, attention manipulation contrast: $F(1,98)=6.83, p=.01, \eta_{\mathrm{p}}^{2}=.065$; working memory contrast: $F(1,98)=.85, p=.36]$. The effect of Factor 2 on P140 was not significant ( $p$ values $>.65$ ).

\section{Discussion}

In this study, we isolated a relatively early ERP (P140) component to evaluate the effects of goal-directed attention on threat processing in psychopathy. The results provided good support for our hypothesis that psychopathy would be associated with superior selective

\footnotetext{
${ }^{3}$ It is common in ERP studies to examine the behavioral results of a task. The Psychopathy $\times$ Threat $\times$ Focus of attention interaction contrast was at trend-level, $F(1,99)=3.21, p=.076$, with individuals high on psychopathy relative to those low on psychopathy responding faster in the threat-focus condition than in the alternative-focus conditions, particularly during presentation of red letters. Notably, this critical interaction contrast was significant in the Newman et al. (2010) paper $(N=125)$. The reduction of the effect to trend-level in the present study most likely reflects the loss of participants (i.e., power). Moreover, previous ERP studies have interpreted significant ERP findings as the necessary factor in interpreting cognitive operations, whereas psychopathy-related behavioral differences are sufficient but not necessary for implying differences in cognitive operations (Kiehl, Hare, McDonald, \& Brink, 1999).
} 
attention (i.e., greater P140) under alternative-focus versus threat-focus conditions. In light of the fact that these findings were obtained under conditions that manipulate precisely the attentional circumstances specified by the response modulation model and tracked the FPS deficit reported by Newman et al. (2010), the present study provides preliminary evidence that psychopathy-related deficits in response modulation are associated with idiosyncrasies at an early stage of selective attention.

The fact that offenders with high psychopathy scores displayed larger P140 responses in the alternative-focus conditions suggests that they were better able to maintain their goaldirected focus and ignore the salient threat of shock associated with red letters. One interpretation of this finding is that, their response modulation deficit reduces competition between goal-relevant and irrelevant stimuli when conditions allow a person to use early selective attention to screen out distraction (i.e., alternative focus conditions). Conversely, when attending to threat-relevant cues was required to perform the task (i.e., threat-focus condition), the emotion information appeared to hamper their goal-relevant response selection (see also Hare, 1978). Thus, psychopathic individuals may be more efficient at focusing attention under alternative focus conditions that allow for early selection, but less adept at maintaining a goal-relevant focus once competing demands (e.g., to process emotion stimuli and select an appropriate response) are registered.

This proposal is consistent with the performance of psychopathic individuals on Stroop and Flanker tasks. In standard versions of the color-word and number Stroop tasks, participants first perceive the conflicting elements and must then reprioritize attention to the appropriate element of the display. Under such conditions, psychopathic individuals show normal levels of interference (Blair et al., 2006; Hiatt et al., 2004). Conversely, on Stroop-like tasks that facilitate early selection of goal-relevant information by spatially or temporally separating the incongruent elements of the display, psychopathic individuals display significantly less interference than nonpsychopathic individuals (Hiatt et al., 2004; Mitchell et al., 2006). Essentially, these tasks alleviate the need for online reprioritization of the conflict because psychopathic individuals are able to establish an early attentional focus that effectively screens out distraction. Similarly, in the present task, the emotion distraction in the alternative-focus conditions, although spatially integrated, is peripheral to the primary task, and does not necessarily prime a conflicting response that must be resolved.

Corroborating this interpretation of the Stroop data, Zeier et al. (2009) used pretrial cuing stimuli so that participants could orient attention to the location of the task-relevant target before presenting the target and distracting flanker stimuli. On other trials, the pretrial cues directed attention to both the target and distractor locations. Whereas psychopathic participants displayed significantly less interference than controls in the early-selection condition, they displayed nonsignificantly more interference in the condition that cued both target and distractor locations. Combined, these studies show that psychopathic participants are significantly less sensitive to information if it is peripheral to their preestablished focus of goal-directed behavior. However, they show little evidence of superior selective attention under conditions that vitiate their penchant for early selection.

The results of the present study complement this theoretical framework and empirical evidence. When participants were instructed to focus on the goal-relevant feature of the experimental stimuli and screen-out the irrelevant distractor (i.e., threat), psychopathic offenders displayed larger P140 responses than when experimental conditions precluded such focusing (i.e., threat-focus condition). Conversely, the P140 responses of offenders with low psychopathy scores were quite similar across threat-focus and alternative-focus conditions. Moreover, in contrast to more psychopathic participants, their P140 to threat (red) letters was descriptively smaller in alternative- focus conditions than in the threat- 
focus condition. Such findings may reflect the fact that their early attention in the threatfocus condition was focused on a single dimension (i.e., threat), but distributed across both the primary task and threat (peripheral, but threat-relevant) information in the alternativefocus conditions. This possibility is consistent with the fact that they displayed descriptively larger P140 to threat (red) letter in threat-focus than alternative-focus conditions (i.e., less P140 in alternative-focus conditions because of attention distribution). Moreover, it is also consistent with the fact that individuals low on psychopathy continued to display fearpotentiated startle under alternative-focus conditions in the Newman et al. (2010) study.

An alternative perspective on the current findings is that individuals with high psychopathy scores were less motivated to track the emotion cues inherent to letter color (Lykken, 1995). In other words, the smaller P140 responses displayed by psychopathic offenders may reflect disengagement in the threat-focus condition, whereas their larger P140 in the alternativefocus conditions may reflect enhanced attention to the goal-relevant features owing to their diminished interest in the peripheral threat cues. Moreover, the fact that the modulation of P140 is specific to red (threat) cues may suggest that the psychopathic-response to threat is weaker overall. Although this alternative explanation is consistent with the view that psychopathy entails a fundamental emotion deficit, it is inconsistent with the fact that psychopathic offenders demonstrated normal FPS under threat-focus conditions in the Newman et al. (2010) study. Moreover, this alternative explanation cannot account for the parallel findings obtained with the Stroop and Flanker tasks. For these and the other reasons that gave rise to our a priori hypothesis, we believe that psychopathic offenders did, in fact, allocate attention to the threat cues in the threat-focus condition and that their early attentional encoding and reaction to the threat cues weakened their goal-directed focus (i.e., P140).

Finally, examination of the PCL-R factors suggests that the early selection effect identified in this study is primarily associated with the affective-interpersonal features (Factor 1) of psychopathy as opposed to the impulsive-antisocial life-style features (Factor 2) of psychopathy. However, other research has demonstrated that the early attention bottleneck also applies to the impulsive-antisocial features of psychopathy (Baskin-Sommers et al., 2011). Thus, further research is needed to clarify the circumstances that determine when attention abnormalities in psychopathy are associated with one or both of the PCL-R factors. Despite this uncertainty regarding the components of psychopathy, there is now growing evidence that the early attention bottleneck is significantly associated with the superordinate PCL-R construct.

One limitation of the current findings is that we cannot provide an unequivocal interpretation of the P140 component used in this study. Our paradigm was designed primarily to measure FPS responses while manipulating the focus of goal-directed behavior rather than to optimize the assessment and interpretation of ERP data. Nonetheless, the timing information provided by the ERP data has yielded clear evidence that the attentional idiosyncrasies associated with psychopathy are apparent at an early stage of processing.

In conjunction with the FPS results (Newman et al., 2010), the present study provides strong support for the central tenet of the response modulation model of psychopathy. As predicted by the model (MacCoon et al., 2004; Patterson \& Newman, 1993), psychopathy is associated with abnormalities in early selective attention, which, in turn, reduce reactivity to peripheral emotion-related cues that normally modulate goal-directed behavior. The results provide evidence that selective attention significantly moderates both the deficits (i.e., FPS; Newman et al., 2010) and the benefits (i.e., attentional focus) associated with psychopathy. On the one hand, their attentional bottleneck allows psychopathic individuals to be more effective at filtering out distraction and rigidly focused on their personal goal. On the other 
hand, however, it may leave them vulnerable to overallocating attention to goal-relevant cues at the expense of processing other context-relevant information. For example, such overfocusing could possibly underlie the self-centered, callous traits associated with psychopathy, particularly Factor 1. More generally, a deficit in the ability to process multiple aspects of information may leave psychopaths' oblivious to the potentially devastating consequences of their behavior. Overall, in light of reliable evidence that attention significantly moderates the inhibitory (Zeier et al., 2009), affective (Newman et al., 2010), and self-regulation deficits associated with psychopathy (Newman \& Lorenz, 2003), it is crucial for future research to specify the cognitive-affective networks responsible for the response modulation deficits of psychopathic individuals.

\section{Acknowledgments}

This work was supported by NIMH Grants 5R01MH078980-03 and T32-MH018931. We thank many at the Wisconsin Department of Corrections and the Dodge Correctional Institution for making this research possible.

\section{References}

Baskin-Sommers AR, Curtin JJ, Newman JP. Specifying the attentional selection that moderates the fearlessness of psychopathic offenders. Psychological Science. 2011; 22:226-234. [PubMed: 21245494]

Blair RJR, Mitchell DVG. Psychopathy, attention and emotion. Psychological Medicine. 2009; 39:543-555. [PubMed: 18700991]

Blair RJR, Sellars C, Strickland I, Clark F, Williams AO, Smith M, Jones L. Emotion attributions in the psychopath. Personality and Individual Differences. 1995; 19:431-437.

Deutsch JA, Deutsch D. Attention: Some theoretical considerations. Psychological Review. 1963; 70:51-61. [PubMed: 13983431]

Driver J. A selective review of selective attention research from the past century. British Journal of Psychology. 2001; 92:53-78.

Duncan J. The locus of interference in the perception of simultaneous stimuli. Psychological Review. 1980; 87:272-300. [PubMed: 7384344]

Flor H, Birbaumer N, Hermann C, Ziegler S, Patrick CJ. Aversive Pavlovian conditioning in psychopaths: Peripheral and central correlates. Psychophysiology. 2002; 39:505-518. [PubMed: 12212643]

Forster KI, Forster JC. DMDX: A Windows display program with millisecond accuracy. Behavioral Research Methods. 2003; 35:116-124.

Forth AE, Hare RD. The contingent negative variation in psychopaths. Psychophysiology. 1989; 26:676-682. [PubMed: 2629015]

Hare, RD. Hare Psychopathy Checklist-Revised (PCL-R). 2nd ed.. Toronto, ON, Canada: MHS; 2003.

Hiatt KD, Schmitt WA, Newman JP. Stroop tasks reveal abnormal selective attention among psychopathic offenders. Neuropsychology. 2004; 18:50-59. [PubMed: 14744187]

Hillyard SA, Vogel EK, Luck SJ. Sensory gain control (amplification) as a mechanism of selective attention: Electrophysiological and neuroimaging evidence. Philosophical Transactions of the Royal Society: Biological Sciences. 1998; 393:1257-1270. [PubMed: 9770220]

Jutai JW, Hare RD. Psychopathy and selective attention during performance of a complex perceptualmotor task. Psychophysiology. 1983; 20:146-151. [PubMed: 6844513]

Jutai JW, Hare RD, Connolly JF. Psychopathy and event-related brain potentials (ERPs) associated with attention to speech stimuli. Personality \& Individual Differences. 1987; 8:175-184.

Kastner S, Ungerleider LG. Mechanisms of visual attention in the human cortex. Annual Review of Neuroscience. 2000; 23:315-341.

Kiehl KA. A cognitive neuroscience perspective on psychopathy: Evidence for paralimbic system dysfunction. Psychiatry Research. 2006; 142:107-128. [PubMed: 16712954] 
Kiehl KA, Bates AT, Laurens KR, Hare RD, Liddle PF. Brain potentials implicate temporal lobe abnormalities in psychopathy. Journal of Abnormal Psychology. 2006; 115:443-453. [PubMed: 16866585]

Kiehl KA, Hare RD, McDonald JJ, Brink J. Semantic and affective processing in psychopaths: An event-related potential (ERP) study. Psychophysiology. 1999; 36:765-774. [PubMed: 10554590]

Kiehl KA, Hare RD, McDonald JJ, Liddle PF. Reduced P3 responses in criminal psychopaths during a visual oddball task. Biological Psychiatry. 1999; 45:1498-1507. [PubMed: 10356633]

Kiehl KA, Smith AM, Hare RD, Liddle PF. An event-related potential investigation of response inhibition in schizophrenia and psychopathy. Biological Psychiatry. 2000; 48:210-221. [PubMed: 10924664]

Lamme VAF, Roelfsema PR. The distinct modes of vision offered by feedforward and recurrent processing. Trends in Neurosciences. 2000; 23:571-579. [PubMed: 11074267]

Lavie N, Hirst A, de Fockert JW, Viding E. Load theory of selective attention and cognitive control. Journal of Experimental Psychology: General. 2004; 133:339-354. [PubMed: 15355143]

Luck, SJ.; Hillyard, SA. The operation of selective attention at multiple stages of processing: Evidence from human and monkey electrophysiology. In: Gazzaniga, MS., editor. The new cognitive neurosciences. 2nd ed.. Cambridge, MA: MIT Press; 1999. p. 687-700.

Lykken, DT. The antisocial personalities. Hillsdale, NJ: Erlbaum; 1995.

MacCoon, DG.; Wallace, JF.; Newman, JP. Self-regulation: The context-appropriate allocation of attentional capacity to dominant and non-dominant cues. In: Baumeister, RF.; Vohs, KD., editors. Handbook of self-regulation: Research, theory, and applications. New York, NY: Guilford Press; 2004. p. 422-446.

Mitchell DGV, Richell RA, Leonard A, Blair RJR. Emotion at the expense of cognition: Psychopathic individuals outperform controls on an operant response task. Journal of Abnormal Psychology. 2006; 115:559-566. [PubMed: 16866596]

Newman, JP. Psychopathic behavior: An information processing perspective. In: Cooke, DJ.; Hare, RD.; Forth, A., editors. Psychopathy: Theory, Research and Implications for Society. The Netherlands: Kluwer Academic Publishers; 1998. p. 81-104.

Newman JP, Curtin JJ, Bertsch J, Baskin-Sommers AR. Attention moderates the fearlessness of psychopathic offenders. Biological Psychiatry. 2010; 67:66-70. [PubMed: 19793581]

Newman, JP.; Lorenz, AR. Response modulation and emotion processing: Implications for psychopathy and other dysregulatory psychopathology. In: Davidson, RJ.; Scherer, K.; Goldsmith, HH., editors. Handbook of affective sciences. Oxford, U. K.: Oxford University Press; 2003. p. 904-929.

Newman, JP.; Baskin-Sommers, AR. Early selective attention abnormalities in psychopathy: Implications for self-regulation. In: Poser, M., editor. Cognitive neuroscience of attention. New York, NY: Guilford Press; (in press).

Patrick CJ. Emotion and psychopathy: Startling new insights. Psychophysiology. 1994; 31:319-330. [PubMed: 10690912]

Patrick CJ, Bradley MM, Lang PJ. Emotion in the criminal psychopath: Startle reflex modulation. Journal of Abnormal Psychology. 1993; 102:82-92. [PubMed: 8436703]

Patterson CM, Newman JP. Reflectivity and learning from aversive events: Toward a psychological mechanism for the syndromes of disinhibition. Psychological Review. 1993; 100:716-736. [PubMed: 8255955]

Raine A, Venables PH. Enhanced P3 evoked potentials and longer P3 recovery times in psychopaths. Psychophysiology. 1988; 25:30-38. [PubMed: 3353482]

Williamson S, Harpur TJ, Hare RD. Abnormal processing of affective words by psychopaths. Psychophysiology. 1991; 28:260-273. [PubMed: 1946892]

Zeier J, Maxwell JS, Newman JP. Attention moderates the processing of inhibitory information in primary psychopathy. Journal of Abnormal Psychology. 2009; 118:554-563. [PubMed: 19685952] 
A.
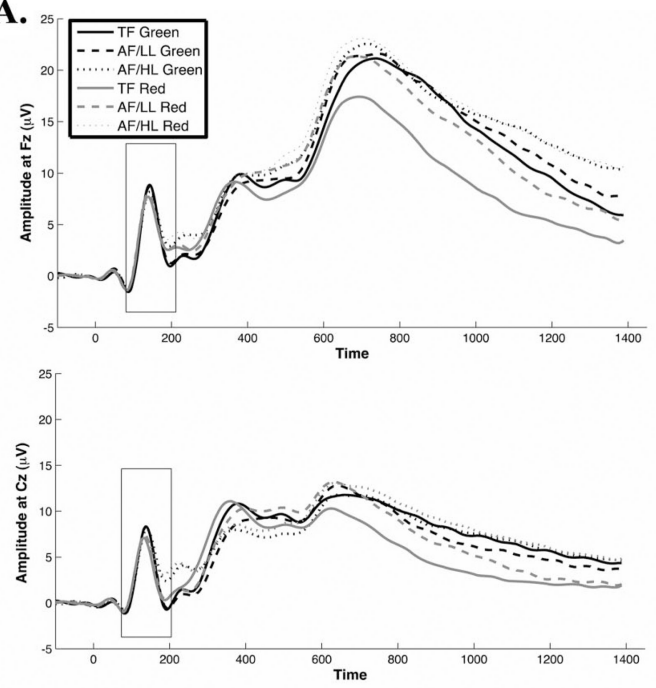

B.
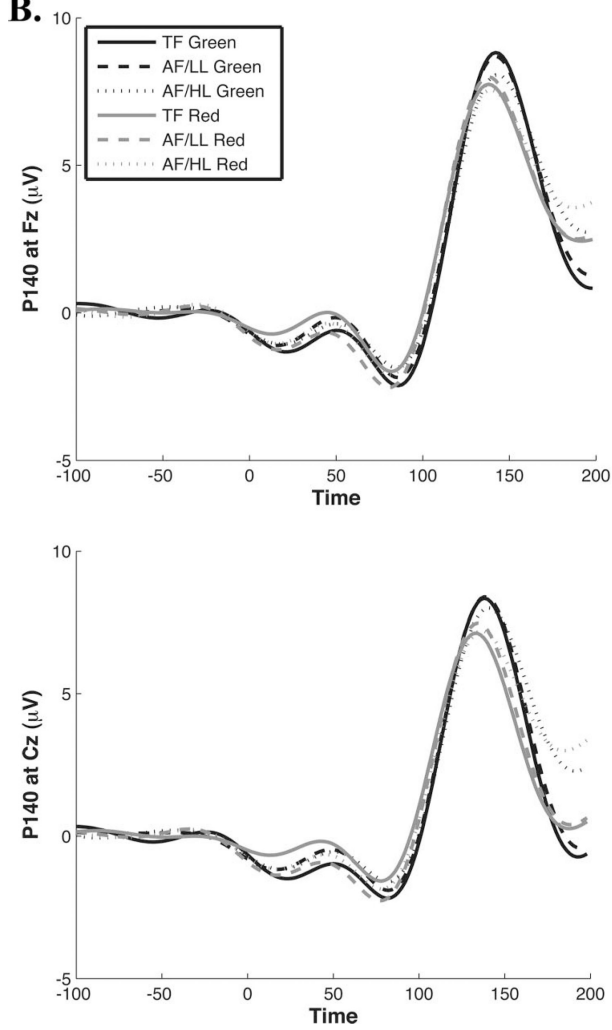
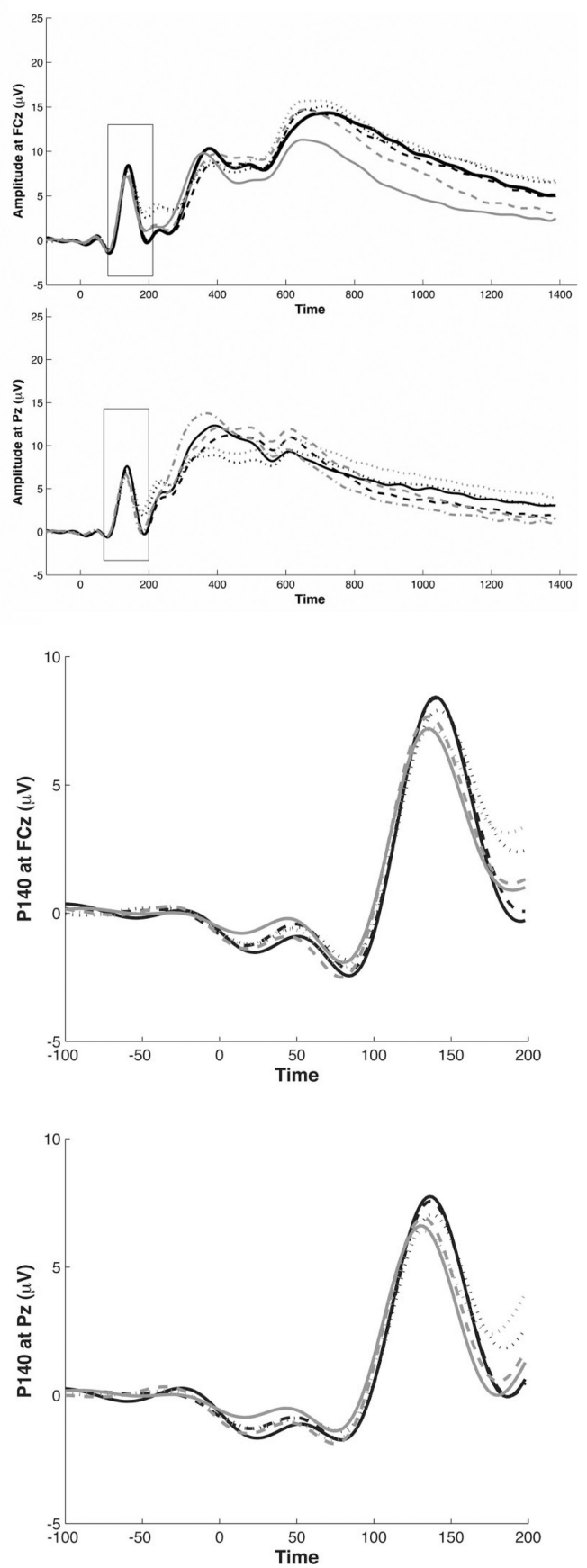

Figure 1.

ERP waveform across midline sites (Fz, FCz, Cz, and Pz). A: Stimulus-locked average ERP waveforms in the three task conditions for all participants at midline electrode sites. A digital low-pass filter was applied offline before plotting the waveforms shown here. Box represents peak identified as P140. B: Isolated P140 waveform in all three task conditions for all participants at midline electrode sites. 


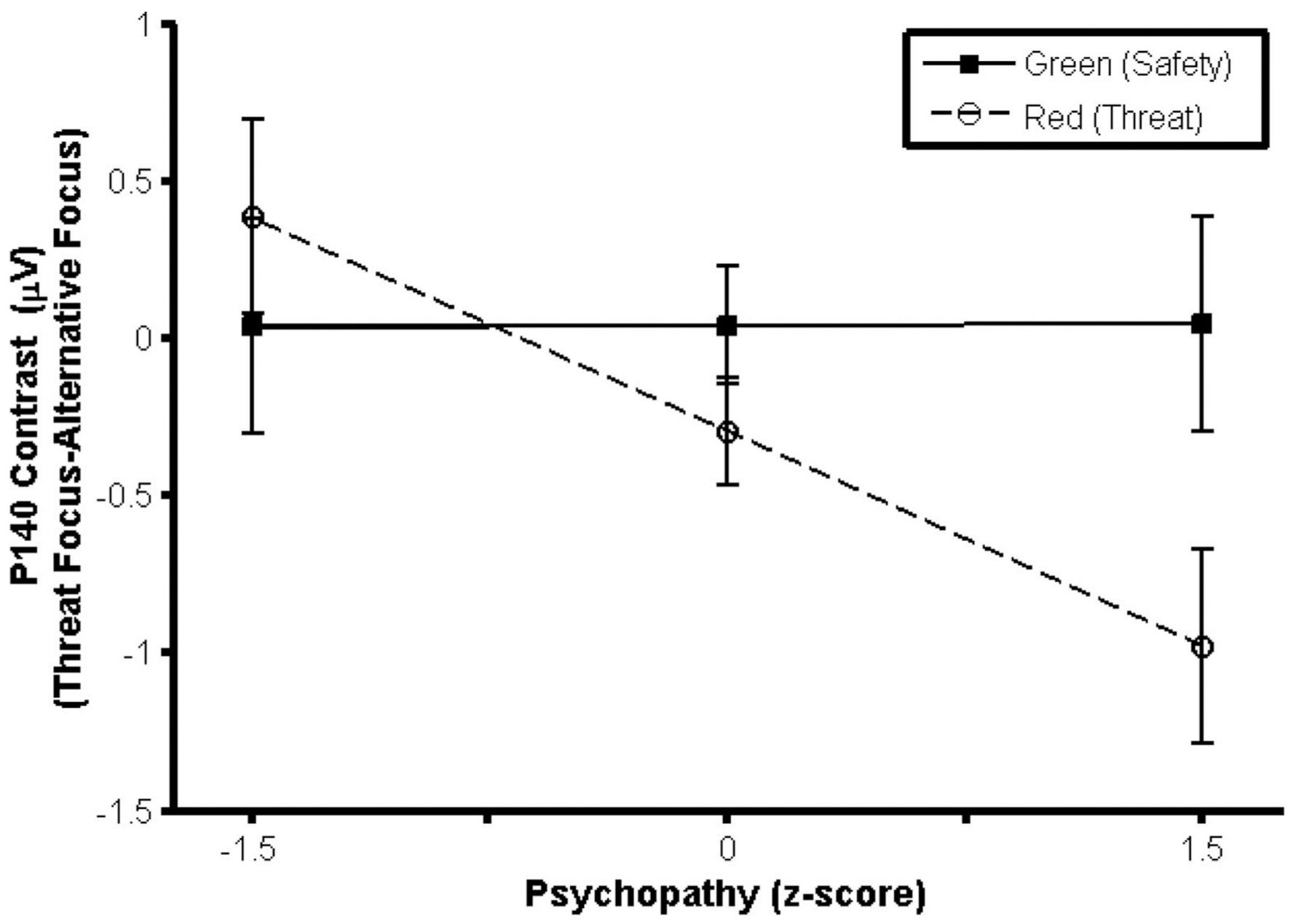

Figure 2.

P140 and psychopathy. The relationship between psychopathy and P140 was moderated by threat and focus of attention (Psychopathy $\times$ Threat $\times$ Attentional focus interaction contrast). Average P140 magnitude was calculated as the mean across midline sites. Using points estimates generated from the GLM, the P140 means for the interaction contrast (attentional manipulation) in the red and green trials displayed for psychopathy were calculated at 1.5 $S D$ below and above the sample mean on psychopathy total scores, respectively. Error bars represent the standard error for the point estimates. The relationship between psychopathy and P140 was moderated by color and focus of attention. There was no psychopathy-related difference in green (no threat) letters, but there was a significant difference in red (threat) letters. Individuals high on psychopathy $(+1.5)$ showed greater P140 to red (threat) letters in alternative-focus versus threat-focus conditions. 


\section{Table 1}

Means and Standard Deviations for Relevant Descriptive Variables $(N=101)$

\begin{tabular}{lrr}
\hline Variable & Mean & SD \\
\hline Demographic & & \\
Age & 30.72 & 7.10 \\
Estimated intelligence & 98.76 & 10.89 \\
Number of violent crimes & 3.20 & 5.30 \\
Number of nonviolent crimes & 16.19 & 23.44 \\
Psychopathy assessment & & \\
PCL-R total score & 23.73 & 6.66 \\
PCL-R factor1 & 8.19 & 3.57 \\
PCL-R factor2 & 13.37 & 3.54 \\
\hline
\end{tabular}

